\title{
Frequency Distribution and Dispersion Curves of Crystal Vibrations of Perdeuterated Polyethylene
}

\author{
Teizo Kitagawa and Tatsuo Miyazawa \\ Institute for Protein Research, Osaka University \\ Kita-ku, Osaka, Japan.
}

(Received May 11, 1970)

\begin{abstract}
Crystal vibrations of perdeuterated polyethylene were treated with interchain as well as intrachain potential functions. Degeneracies and compatibility relations of frequency dispersion curves were derived from irreducible representations of $\boldsymbol{k}$ groups. Frequency dispersion curves for boundaries of the first Brillouin zone were calculated and were assigned to symmetry classes of $\boldsymbol{k}$ groups on the basis of eigenvectors in symmetry co-ordinate space. Theoretical dispersion curves for the phase difference vector along the $c$ axis agree closely with experimental data derived from coherent inelastic scattering of neutrons measured by Feldkamp, et al. The frequency distribution of crystal vibrations was calculated, in agreement with the experimental distribution derived from incoherent scattering of neutrons measured by Lynch, et al.

KEY WORDS Perdeuterated Polyethylene / Crystal Vibrations / Frequency Distribution / Frequency Dispersion Curve / Neutron Scattering / Normal Vibrations.
\end{abstract}

A group-theoretical method for treating lattice vibrations of orthorhombic polyethylene was worked out previously and, with irreducible representations of $\boldsymbol{k}$ groups, Cartesian symmetry co-ordinates of carbon atoms were constructed from translational-symmetry co-ordinates with non-integral index vectors. ${ }^{1} \quad$ Acoustic vibrations in the lowest frequency region are responsible for specific heat in the lowest temperature region. Accordingly a practical method was derived for calculating the frequency distribution of acoustic vibrations from the volume enclosed in constant-frequency surfaces in the three-dimensional phase-difference space. ${ }^{2}$ The frequency distribution of optical as well as acoustic branches is responsible for specific heat in the intermediate temperature region. An efficient method was worked out for calculating the frequency distribution of chain-polymer crystals from vibrational frequencies associated with a number of phase-difference vectors in the first Brillouin zone. ${ }^{2}$

Elastic and inelastic scattering cross sections of thermal neutrons are due to the frequency distribution of crystal vibrations and vibrational displacements of scattering nuclei. For analyses of experimental data of neutron-scattering from orthorhombic polyethylene, the frequency distribution ${ }^{4}$ and scattering cross sections $\mathrm{s}^{5,6}$ were calculated and vibrational assignments were made of inelastic scattering peaks.

Inelastic scattering of thermal neutrons was also measured for the orthorhombic form of perdeuterated polyethylene. ${ }^{7}$ For an oriented sample, coherent inelastic scattering was measured and some dispersion relations were experimentally studied. ${ }^{8}$ Accordingly, for analyzing neutron scattering data of perdeuterated polyethylene, crystal vibrations were treated and the frequency distribution, frequency dispersion curves, their degeneracy and compatibility relations were treated in the present study.

\section{CRYSTAL STRUCTURE AND FORCE CONSTANTS}

The crystal structure of orthorhombic polyethylene was analyzed by Bunn ${ }^{9}$ with the $\mathrm{X}$-ray diffraction method. The space group is $\mathrm{P}$ nam $\left(\mathrm{D}_{2 h}^{16}\right)$ and there are four methylene groups per unit cell, and two molecular chains pass through a unit cell along the $c$ axis (Figure 1). However, the setting angle $(\theta)$ of the skeletal plane with respect to the a axis is not yet 


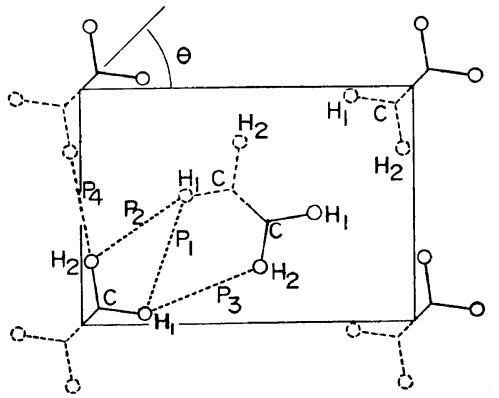

Figure 1. Crystal structure ${ }^{9}$ and inter-hydrogen contacts $\left(\boldsymbol{P}_{1}-\boldsymbol{P}_{4}\right)^{17}$ of orthorhombic polyethylene.

established by X-ray crystal analyses; thus the setting angle at room temperature was reported as $\theta=48.7^{\circ} \quad\left(\right.$ Bunn $\left.^{9}\right), \quad \theta=42^{\circ} \quad\left(\right.$ Smith $^{10}$ for orthorhombic $n$ - $\mathrm{C}_{23} \mathrm{H}_{48}$ ), $\theta=41^{\circ} 23^{\prime}$ (Shearer and Vand ${ }^{11}$ for monoclinic $n-\mathrm{C}_{36} \mathrm{H}_{74}$ ) or $\theta=47.7^{\circ}$ (Teare $^{12}$ for orthorhombic $n-\mathrm{C}_{36} \mathrm{H}_{74}$ ). Snyder ${ }^{13}$ measured the relative infrared intensities of the $\mathbf{B}_{1 u}$ and $\mathbf{B}_{2 u}$ components of the $\mathrm{CH}_{2}$ rocking bands at $-180^{\circ} \mathrm{C}$ and reported the value of $\theta=42^{\circ}$. The setting angle possibly depends upon temperature. In the present study on crystal vibrations at low temperatures, the lattice constants ${ }^{14}$ at $77^{\circ} \mathrm{K}$ were used $\left(a_{0}=7.155\right.$, $b_{0}=4.899$, and $c_{0}=2.547 \AA$ ) and the setting angle was taken as $\theta=45^{\circ}$. As the intramolecular parameters, the bond lengths of $r(\mathrm{C}-\mathrm{C})$ $=1.54$ and $r(\mathrm{C}-\mathrm{H})=1.09 \AA$ and bond angles of $\phi(\mathrm{C}-\mathrm{C}-\mathrm{C})=111^{\circ} 47^{\prime}$ and $\phi(\mathrm{H}-\mathrm{C}-\mathrm{H})$ $=109^{\circ} 27^{\prime}$ were used.

As for the intrachain potential function, the force constants refined by Schachtschneider and Snyder ${ }^{15}$ were used. However, the force constant for the internal rotation about $\mathrm{C}-\mathrm{C}$

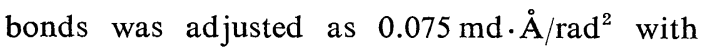
reference to the specific heat ${ }^{16}$ at $\sim 60^{\circ} \mathrm{K}$. As the interchain potential, four types $P_{1}-P_{4}$ in Figure 1) of $\mathrm{H} . \mathrm{H}$ interaction terms ${ }^{17}$ were incorporated. The $\mathrm{H} . \mathrm{H}$ force constants ${ }^{4}$ of $P_{1}=0.004_{2}, \quad P_{2}=0.010_{1}, P_{3}=0.006_{4}$ and $P_{4}=$ $0.011_{2} \mathrm{md} / \AA$ yield the splitting of the infrared active $\mathrm{CH}_{2}$ vibrations as $\Delta \nu$ (bend) $=10$ and $\Delta \nu($ rock $)=10 \mathrm{~cm}^{-1}$ as compared with the experimental values ${ }^{13}$ of $\Delta \nu$ (bend $)=13$ and $\Delta \nu($ rock $)=13 \mathrm{~cm}^{-1}$.

\section{SYMMETRY CO-ORDINATES}

Crystal vibrations are specified with phasedifferences of vibrational displacements between corresponding atoms in adjacent unit cells. For the orthorhombic crystal of polyethylene, there are twelve atoms per unit cell and accordingly there are thirty-six Cartesian symmetry coordinates $[\boldsymbol{S}(\boldsymbol{\delta})]$ associated with any given phasedifference vector $(\boldsymbol{\delta})$. The symmetry co-ordinates of hydrogen atoms may be constructed with the same transformation as the symmetry co-ordinates of carbon atoms ${ }^{1}$, since hydrogen and carbon atoms of orthorhombic polyethylene are group-theoretically on the same special site (the mirror plane perpendicular to the $c$ axis).

The symmetry-co-ordinate subvectors $\boldsymbol{S}_{j}(\boldsymbol{\delta})$ may now be defined as

$$
\boldsymbol{S}_{j}(\boldsymbol{\delta})^{\dagger}=\left[\begin{array}{lll}
\boldsymbol{S}_{j} \mathrm{H}_{1}(\boldsymbol{\delta}) & \boldsymbol{S}_{j} \mathrm{H}_{2}(\boldsymbol{\delta}) & \mathbf{S}_{j} \mathrm{C}(\boldsymbol{\delta})
\end{array}\right]
$$

where the superscripts $\left(\mathrm{H}_{1}, \mathrm{H}_{2}\right.$ and $\left.\mathrm{C}\right)$ characterize atoms (Figure 1) and the subscripts $(j)$ denote symmetry co-ordinates as given in eq 26 of ref 1 . Then, corresponding to eq 36 of ref 1 , the Cartesian symmetry-co-ordinate vector $\boldsymbol{S}(\boldsymbol{\delta})$, with thirty-six components, may be written as

$$
\begin{aligned}
\boldsymbol{S}(\boldsymbol{\delta})^{\dagger}= & {\left[\boldsymbol{S}_{1}(\boldsymbol{\delta})^{\dagger} \boldsymbol{S}_{2}(\boldsymbol{\delta})^{\dagger} \boldsymbol{S}_{3}(\boldsymbol{\delta})^{\dagger} \boldsymbol{S}_{4}(\boldsymbol{\delta})^{\dagger}\right.} \\
& \boldsymbol{S}_{5}(\boldsymbol{\delta})^{\dagger} \boldsymbol{S}_{6}(\boldsymbol{\delta})^{\dagger} \boldsymbol{S}_{8}(\boldsymbol{\delta})^{\dagger} \boldsymbol{S}_{9}(\boldsymbol{\delta})^{\dagger} \\
& \left.\boldsymbol{S}_{7}(\boldsymbol{\delta})^{\dagger} \boldsymbol{S}_{11}(\boldsymbol{\delta})^{\dagger} \boldsymbol{S}_{12}(\boldsymbol{\delta})^{\dagger} \boldsymbol{S}_{10}(\boldsymbol{\delta})^{\dagger}\right]
\end{aligned}
$$

Similarly, for the $i$-th methylene group of a unit cell, the Cartesian co-ordinate vector $\boldsymbol{X}\left(\boldsymbol{\rho}_{i}\right)$, with nine components, may be defined as

$$
\boldsymbol{X}\left(\boldsymbol{\rho}_{i}\right)^{\dagger}=\left[\boldsymbol{x}\left(\boldsymbol{\rho}_{i}\right)^{\dagger} \boldsymbol{y}\left(\boldsymbol{\rho}_{i}\right)^{\dagger} \boldsymbol{z}\left(\boldsymbol{\rho}_{i}\right)^{\dagger}\right]
$$

and

$$
\boldsymbol{\alpha}\left(\boldsymbol{\rho}_{i}\right)^{\dagger}=\left[\alpha^{\mathrm{H}_{1}}\left(\boldsymbol{\rho}_{i}\right) \alpha^{\mathrm{H}_{2}}\left(\boldsymbol{\rho}_{i}\right) \alpha \mathrm{C}\left(\boldsymbol{\rho}_{i}\right)\right]
$$

where $\alpha^{\pi}\left(\boldsymbol{\rho}_{i}\right)$ is the component $\alpha(=x, y$ or $z)$ of the Cartesian co-ordinate vector of the atom $\kappa\left(=\mathrm{H}_{1}, \mathrm{H}_{2}\right.$ or $\left.\mathrm{C}\right)$ and $\boldsymbol{\rho}_{i}$ is the non-integral index vector of the $i$-th methylene group as given in eq 1 of ref 1 . Accordingly, the elements $\left(c_{i}, s_{i}\right.$, and 0$)$ of the transformation submatrices $\left(\boldsymbol{u}_{i}{ }^{c}\right.$ and $\left.\boldsymbol{u}_{i}{ }^{s}\right)$ of eq 38 of ref 1 need to be substituted with three-dimensional constant matrices, 
Crystal Vibrations and Dispersion Curves of Perdeuterated Polyethylene

$$
\begin{gathered}
c_{i} \longrightarrow\left[\begin{array}{lll}
c_{i} & 0 & 0 \\
0 & c_{i} & 0 \\
0 & 0 & c_{i}
\end{array}\right] \\
s_{i} \longrightarrow\left[\begin{array}{lll}
s_{i} & 0 & 0 \\
0 & s_{i} & 0 \\
0 & 0 & s_{i}
\end{array}\right] \\
0 \longrightarrow\left[\begin{array}{lll}
0 & 0 & 0 \\
0 & 0 & 0 \\
0 & 0 & 0
\end{array}\right]
\end{gathered}
$$

Finally, eq 37 of ref 1 applies for the transformation of the $\boldsymbol{X}\left(\boldsymbol{\rho}_{1}\right), \boldsymbol{X}\left(\boldsymbol{\rho}_{2}\right), \boldsymbol{X}\left(\boldsymbol{\rho}_{3}\right)$ and $\boldsymbol{X}\left(\boldsymbol{\rho}_{4}\right)$ vectors [eq 3 ] into the Cartesian symmetryco-ordinate vector $\boldsymbol{S}(\boldsymbol{\delta})$ [eq 2 ].

\section{COMPATIBILITY}

For special 8 vectors (with components of $\delta=0$ or $\pi$ ), dynamical matrices of crystal vibrations may be factored and frequencydispersion curves may be assigned to symmetry species of the $\boldsymbol{k}$ group. ${ }^{18}$ However, vibrational frequencies change continuously with $\delta$ and the symmetry co-ordinates for a special $\boldsymbol{\delta}$ vector can serve as symmetry co-ordinates for $\boldsymbol{k}$ groups of lower symmetry (see Table 12 of ref 1). These compatibility relations are derived from irreducible representation of $\boldsymbol{k}$ groups.

For the space group $\mathbf{P}$ nam, the compatibility relations of frequency-dispersion curves were derived as shown in Table I, where symbols follow the BSW notation. ${ }^{20}$ The two symmetry

Table I. Compatibility relations of frequency-dispersion curves of polyethylene crystal (P nam)

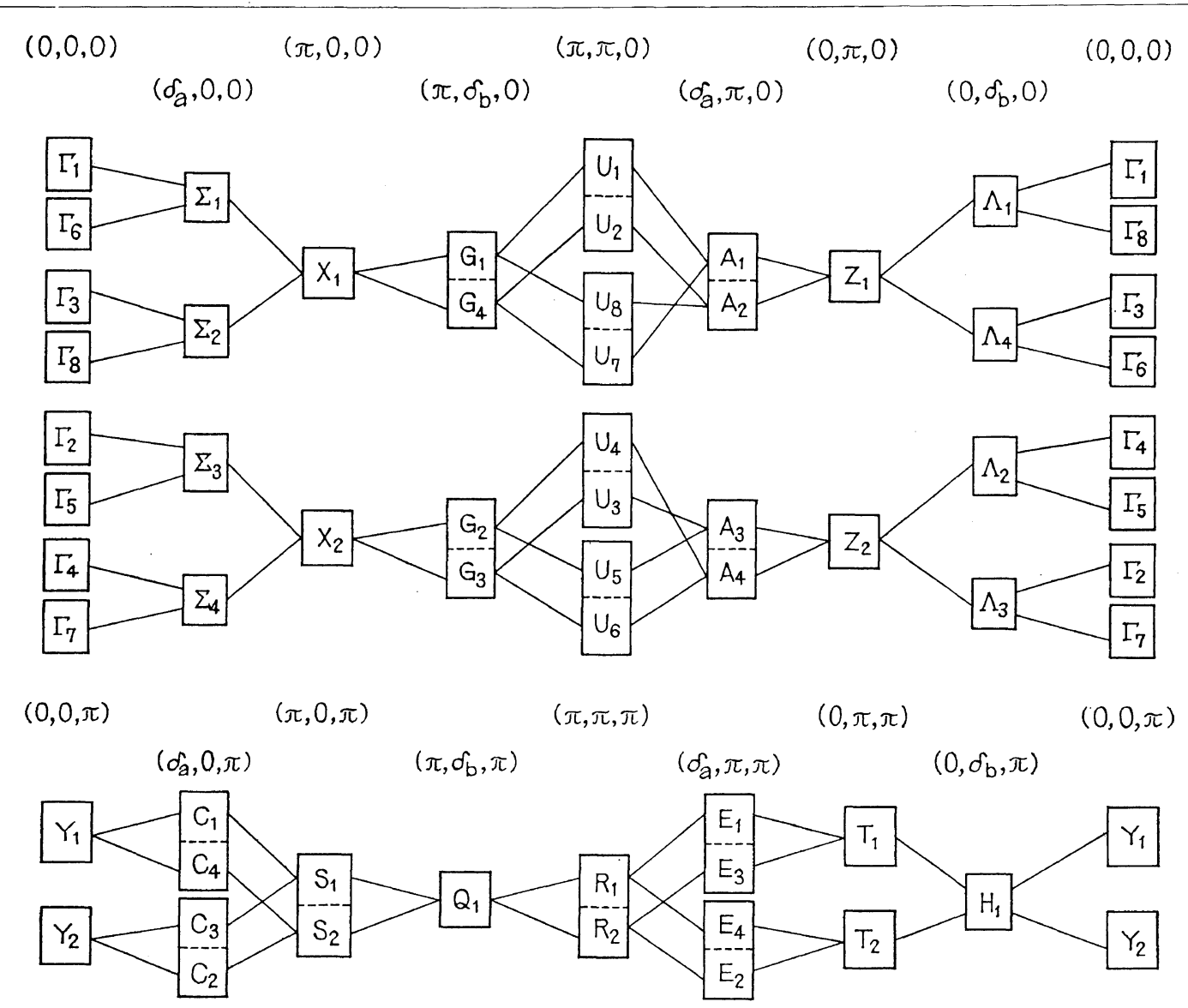


Table I. Continued

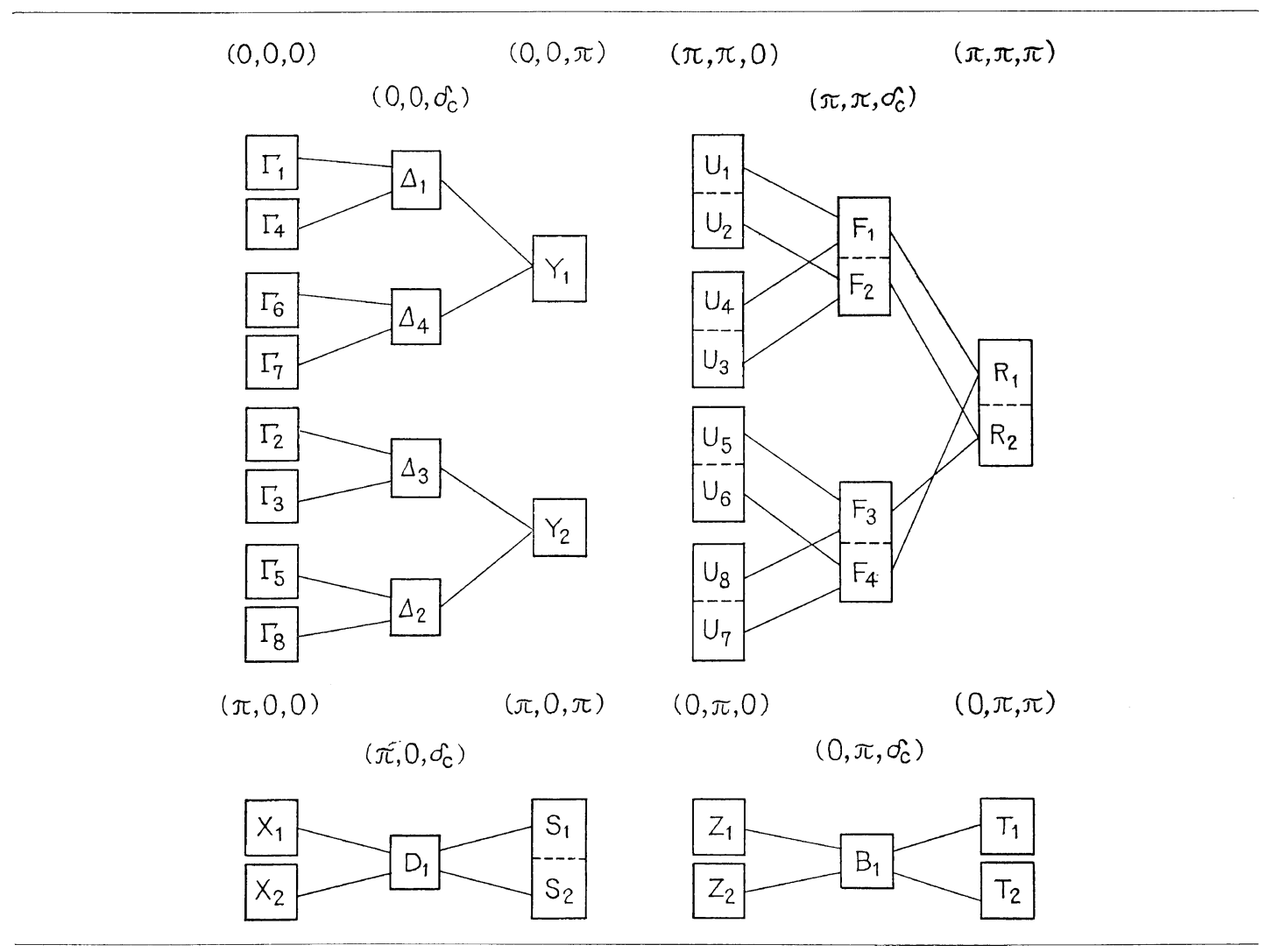

species partitioned by a broken line in a block are degenerate pairs due to the time reversal symmetry, and accordingly the degeneracy of frequency dispersion curves is twice the degeneracy due to the space symmetry. The time reversal degeneracy $\left(t_{s}\right)$ and space degeneracy $\left(f_{s}\right)$ were derived previously (Table 10 of ref 1$)$.

\section{DISPERSION CURVES}

Dynamical matrices of perdeuterated polyethylene $\left(-\mathrm{CD}_{2}\right)_{p}$ for various \& vectors were constructed on the basis of the Cartesian symmetry co-ordinate vector of eq 2 and were diagonalized. Frequency dispersion curves of crystal vibrations below $500 \mathrm{~cm}^{-1}$ are shown in Figures $3 a-h$. Since the dynamical matrix may be factored into blocks of symmetry species of the $\boldsymbol{k}$ group, the dispersion curves of different

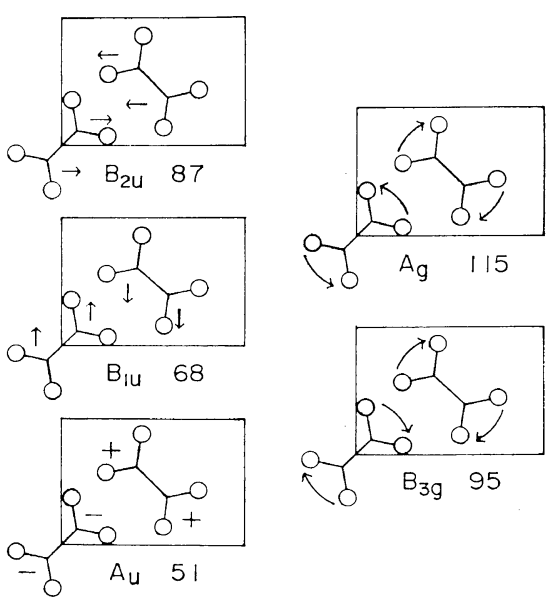

Figure 2. Vibrational modes and calculated frequencies of lattice vibrations of the orthorhombic form of perdeuterated polyethylene. 
species may intersect each other. Those of the same species, however, do not intersect each other.

$\Gamma$ Point. At the origin $\Gamma(0,0,0)$ of the Brillouin zone, the $\boldsymbol{k}$ group is $\mathrm{D}_{2 h}$, the factor group of the space group. The vibrational modes and calculated frequencies of the five interchain vibrations are shown in Figure 2. The $\mathrm{B}_{1 u}\left(\Gamma_{6}\right)$ and $\mathrm{B}_{2 u}\left(\Gamma_{8}\right)$ vibrations are infrared active while the $\mathrm{A}_{g}\left(\Gamma_{1}\right)$ and $\mathrm{B}_{3 g}\left(\Gamma_{3}\right)$ vibrations are Raman active. The $\mathrm{B}_{1 u}$ vibration calculated

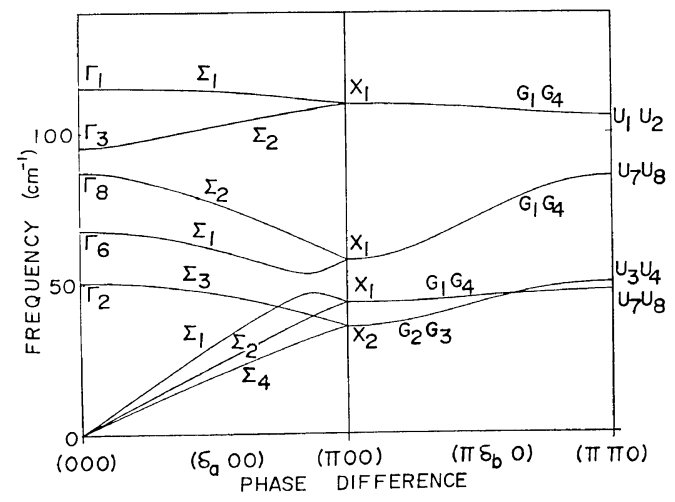

(a)

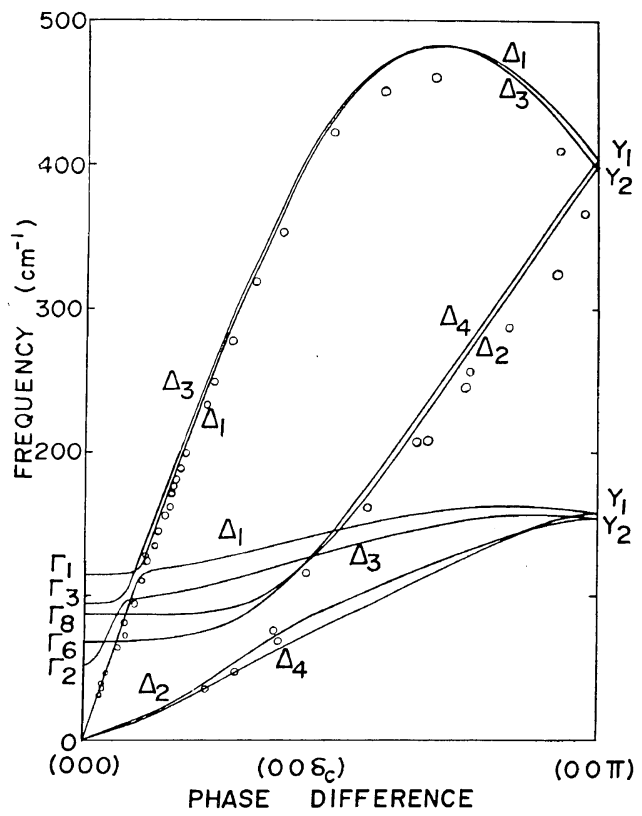

(c) at $68 \mathrm{~cm}^{-1}$ was, in fact, observed ${ }^{21}$ at $66.7 \mathrm{~cm}^{-1}$ for $\mathrm{C}_{100} \mathrm{D}_{202}$.

$\Sigma$ and 1 Points. At the $\Sigma\left(\delta_{a}, 0,0\right)$ and $\Lambda\left(0, \delta_{b}, 0\right)$ points, the $k$ group is $\mathrm{C}_{2 v}$, with four symmetry species $\left(\Sigma_{1}-\Sigma_{4}\right.$ and $\left.\Lambda_{1}-\Lambda_{4}\right)$. Dispersion curves are shown in Figures $3 a$ and $b$.

The highest $\Sigma_{1}$ and $\Sigma_{2}$ (also $\Lambda_{1}$ and $\Lambda_{4}$ ) vibrations are due to rotational modes about the chain axis and the next highest $\Sigma_{1}$ and $\Sigma_{2}$ (also $\Lambda_{1}$ and $\Lambda_{4}$ ) vibrations are due to translational modes in the $c$ plane. The $\Sigma_{3}$ and $\Sigma_{4}$ (also $\Lambda_{2}$

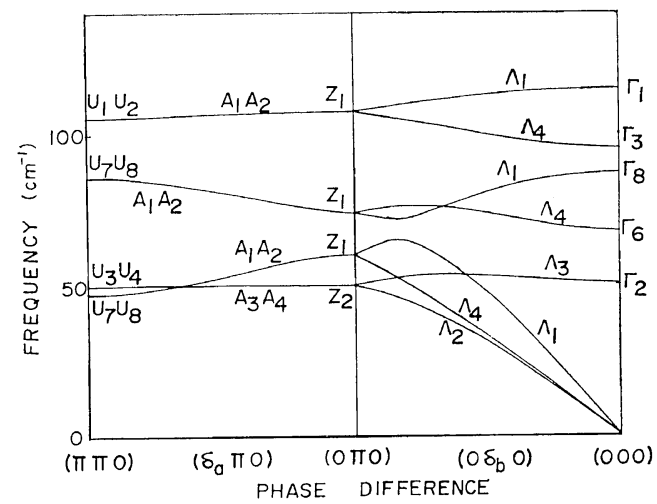

(b)

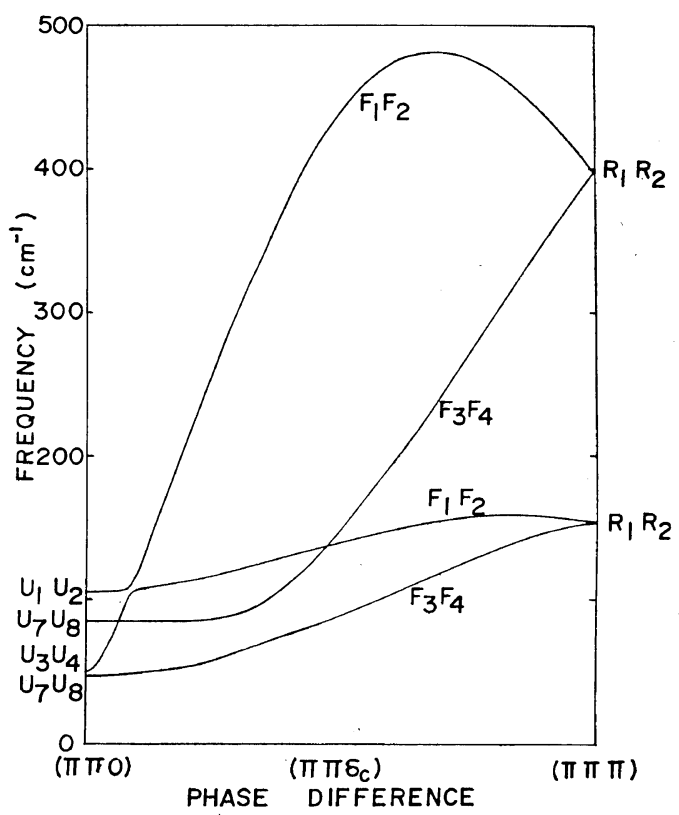

(d)

Figure 3. Frequency-dispersion curves of the orthorhombic form of perdeuterated polyethylene in the region below $500 \mathrm{~cm}^{-1}$. 


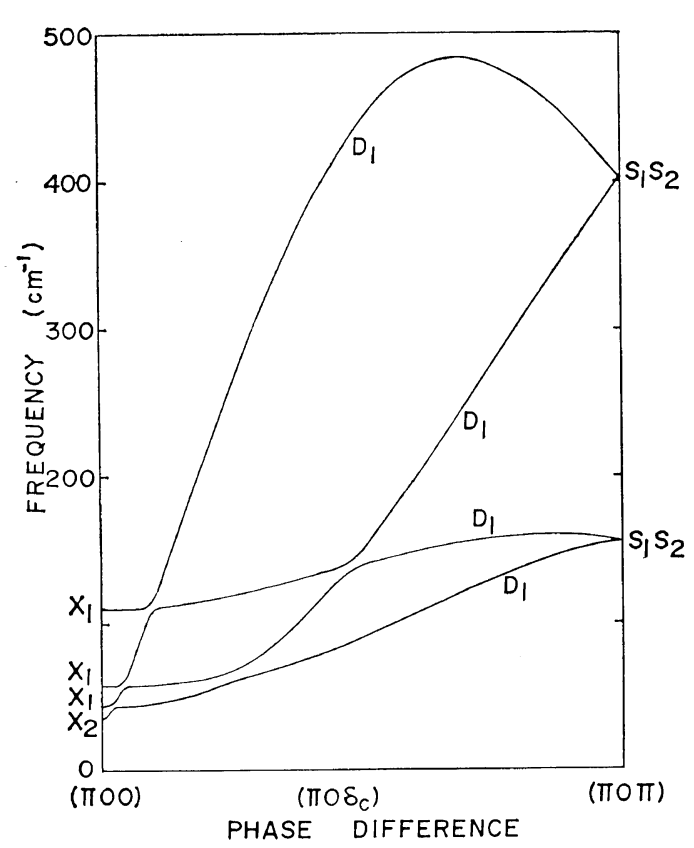

(e)

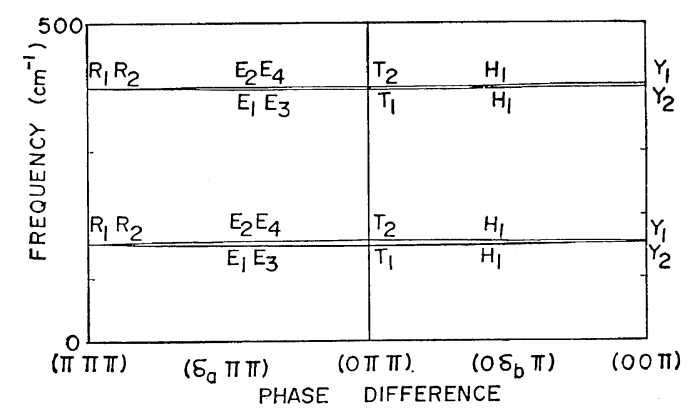

(g)

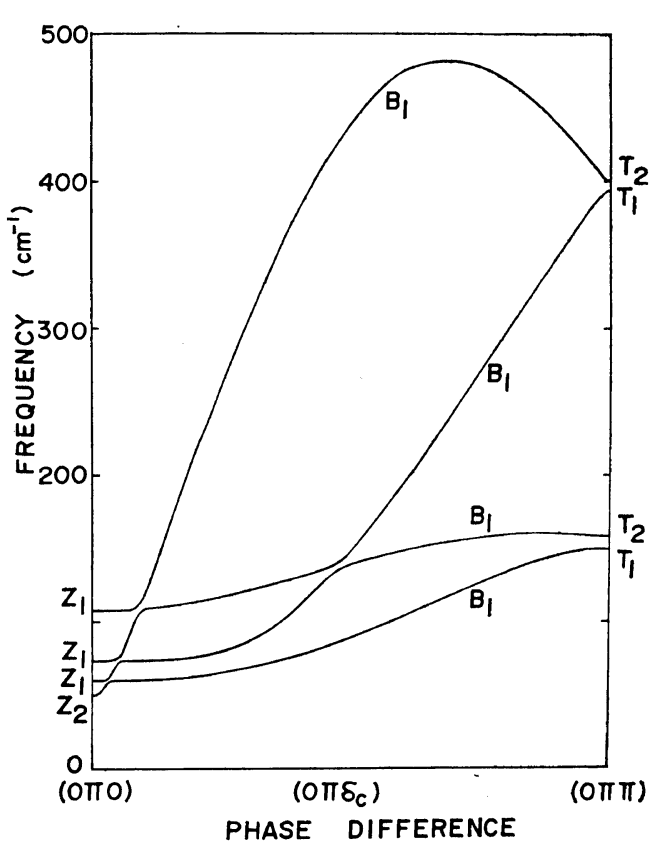

(f)

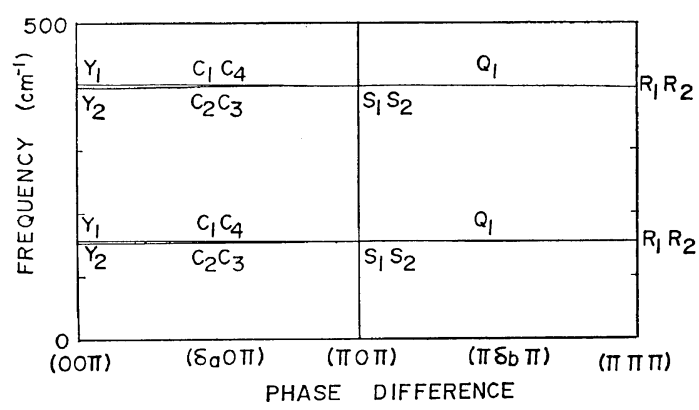

(h)

Figure 3. (continued).

and $\Lambda_{3}$ ) vibrations are due to translational modes along the $c$ axis.

Acoustic modes of the lowest-frequency branches $\left(\Sigma_{1}, \Sigma_{2}, \Sigma_{4} ; \Lambda_{1}, \Lambda_{4}, \Lambda_{2}\right)$ are classified into longitudinal and transverse modes. The $\Sigma_{1}$ and $\Lambda_{1}$ branches are longitudinal and their inclinations are given by elastic constants; $\left(\partial \nu / \partial \delta_{a}\right)_{0}=$ $\left(c_{11} / \rho\right)^{1 / 2} / 2 \pi a_{0} c$, and $\left(\partial \nu / \partial \delta_{b}\right)_{0}=\left(c_{22} / \rho\right)^{1 / 2} / 2 \pi b_{0} c$, where $\rho$ is the density, $c$ is the velocity of light, and $a_{0}$ and $b_{0}$ are lattice constants. The $\Sigma_{2}$ and $\Sigma_{4}$ branches are transverse and are associated with the translatory modes along the $b$ and $c$ axes; $\left(\partial \nu / \partial \delta_{a}\right)_{0}=\left(c_{66} / \rho\right)^{1 / 2} / 2 \pi a_{0} c$ and $\left(c_{55} / \rho\right)^{1 / 2} / 2 \pi a_{0} c$, respectively. The $\Lambda_{4}$ and $\Lambda_{2}$ branches are also transverse and are associated with the translatory modes along the $a$ and $c$ axes: $\left(\partial \nu / \partial \delta_{b}\right)_{0}=\left(c_{66} / \rho\right)^{1 / 2} / 2 \pi b_{0} c$ and $\left(c_{44} / \rho\right)^{1 / 2} / 2$ $\pi b_{0} c$, respectively.

As the phase difference $\delta_{a}$ approaches $\pi$, the $\Sigma_{1}$ and $\Sigma_{2}$ (or $\Sigma_{3}$ and $\Sigma_{4}$ ) branches approach each other and finally meet at the zone edge of $(\pi, 0,0)$, to form doubly-degenerate $X_{1}$ (or $X_{2}$ ) vibrations. The inclinations $\left(\partial \nu / \partial \delta_{a}\right)_{\pi}$ of the $\Sigma_{1}$ and $\Sigma_{2}$ (or $\Sigma_{3}$ and $\Sigma_{4}$ ) branches are of same 


\section{Crystal Vibrations and Dispersion Curves of Perdeuterated Polyethylene}

magnitude as, but of opposite sign to, each other. Also at the zone edge of $(0, \pi, 0)$, the $\Lambda_{1}$ and $\Lambda_{4}$ (or $\Lambda_{3}$ and $\Lambda_{2}$ ) branches meet to form doubly-degenerate $Z_{1}$ (or $Z_{2}$ ) vibrations.

$\triangle$ Point. Dispersion curves for the $\Delta\left(0,0, \delta_{c}\right)$ points were published previously by Tasumi and Krimm. ${ }^{22}$ In the present study, however, symmetry assignments were made. At the $\Delta$ points, the $\boldsymbol{k}$ group is $\mathrm{C}_{2 v}$, with four symmetry species $\left(A_{1}-\Delta_{4}\right)$. Dispersion curves are shown in Figure 3c. As the phase-difference changes from $\delta_{c}=0$ to $\pi$, interchain vibrations turn into intrachain vibrations and accordingly vibrational frequencies vary sensitively with $\delta_{c}$.

Except for small values of $\delta_{c}$, the higher $\Delta_{1}$ and $\Delta_{3}$ branches are largely due to $\mathrm{C}-\mathrm{C}-\mathrm{C}$ bending modes. If there were no interchain interactions, there should be a longitudinal branch of the isolated chain, starting from $\nu=$ 0 at the $\Gamma$ point. Actually, however, because of interchain interactions, the longitudinal branch is split into the $\Delta_{1}$ and $\Delta_{3}$ branches of the crystal. The lower $\Delta_{1}$ and $\Delta_{3}$ branches are largely due to internal-rotation modes about $\mathrm{C}-\mathrm{C}$ bonds.

Recently, frequency-dispersion relations of perdeuterated polyethylene were experimentally studied from measurements of coherent inelastic scattering of thermal neutrons. ${ }^{8}$ The scattering vector was parallel to the $c$ axis and accordingly inelastic scattering cross sections were proportional to squared displacements of scattering nuclei along the $c$ axis. Inelastic scattering due to internal-rotation vibrations was hardly detected. As shown in Figure 3c, observed frequency-dispersion relations (open circles) agree fairly well with calculated relations (solid lines). The splitting of the $\Delta_{1}$ and $\Delta_{3}$ branches or of the $\Delta_{2}$ and $\Delta_{4}$ branches was not detected possibly because of the low resolution of scattering measurements. The maximum frequency of the higher $\Delta_{1}$ and $\Delta_{3}$ branches is observed at $452 \mathrm{~cm}^{-1}$, slightly lower than the calculated frequency of $480 \mathrm{~cm}^{-1}$. However, Raman lines due to accordion vibrations of $n-\mathrm{C}_{94} \mathrm{H}_{190}$ were observed $^{23}$ at 536 and $556 \mathrm{~cm}^{-1}$, suggesting the maximum frequency of perdeuterated polyethylene higher than $470 \mathrm{~cm}^{-1}$.

$X, Z$ and $Y$ Points. At the $X(\pi, 0,0), Z(0, \pi, 0)$ and $Y(0,0, \pi)$ points, the $k$ group is $\mathrm{D}_{2 h}$. Irreducible representations are two-dimensional and crystal vibrations are doubly degenerate, yielding four different frequencies in the region below $500 \mathrm{~cm}^{-1}$ (Figures $3 \mathrm{a}, \mathrm{b}$ and $\mathrm{c}$ ). The symmetry co-ordinates for the $X$ point were discussed previously. ${ }^{1}$

Inclinations of the dispersion curves at the $Z$ point may be seen in Figure $3 \mathrm{~b}:\left(\partial \nu / \partial \delta_{a}\right)_{0}=0$ but $\left(\partial \nu / \partial \delta_{b}\right)_{\pi} \neq 0$. Generally at an edge of the irreducible zone of orthorhombic crystals, the inclinations of dispersion curves are always $(\partial \nu / \partial \delta)=0$ if, the four-fold or two-fold degeneracy persists, but are $(\partial \nu / \partial \delta) \neq 0$ if the degeneracy is reduced.

$G, A$ and $U$ Points. For the $G\left(\pi, \delta_{b}, 0\right)$ and $A\left(\delta_{a}, \pi, 0\right)$ points, the $\boldsymbol{k}$ group is $\mathrm{C}_{2 v}$ but for the $U(\pi, \pi, 0)$ point the $\boldsymbol{k}$ group is $\mathrm{D}_{2 h}$. However, for all these points, irreducible representations are one-dimensional. The time-reversal degeneracy appears (Table 10 of ref 1), and symmetry species form degenerate pairs: $\left(G_{1}\right.$ and $\left.G_{4}\right),\left(G_{2}\right.$ and $\left.G_{3}\right),\left(A_{1}\right.$ and $\left.A_{2}\right),\left(A_{3}\right.$ and $\left.A_{4}\right),\left(U_{1}\right.$ and $\left.U_{2}\right),\left(U_{3}\right.$ an $\left.U_{4}\right),\left(U_{5}\right.$ and $\left.U_{6}\right)$ and $\left(U_{7}\right.$ and $U_{8}$ ). Four dispersion curves are shown in Figures $3 \mathrm{a}$ and $\mathrm{b}$. The symmetry co-ordinates for the $U$ point were discussed previously. ${ }^{1}$

$F$ and $R$ Points. For the $F\left(\pi, \pi, \delta_{c}\right)$ point, the $\boldsymbol{k}$ group is again $\mathrm{C}_{2 v}$ and irreducible representations are one-dimensional. Because of the timereversal degeneracy, symmetry species form degenerate pairs $\left(F_{1}\right.$ and $\left.F_{2}\right)$ and $\left(F_{3}\right.$ and $\left.F_{4}\right)$. Dispersion curves are shown in Figure $3 \mathrm{~d}$. Vibrational frequencies change sensitively with the phase-difference $\left(\delta_{c}\right)$ along the chain axis.

Finally, at the $R(\pi, \pi, \pi)$ point, the $\boldsymbol{k}$ group is $\mathrm{D}_{2 h}$, with the time-reversal degeneracy. Irreducible representations are two-dimensional and accordingly crystal vibrations are quadruply degenerate. Symmetry co-ordinates for the $R$ point were discussed previously. ${ }^{1}$

$D$ and $B$ Points. For the $D\left(\pi, 0, \delta_{c}\right)$ and $B\left(0, \pi, \delta_{c}\right)$ points, the $\boldsymbol{k}$ group is $\mathrm{C}_{2 v}$. There is only one symmetry species with the two-dimensional representation and accordingly crystal vibrations are doubly degenerate. Dispersion relations for the $D$ (Figure $3 \mathrm{e}$ ) and $B$ (Figure $3 \mathrm{f}$ ) points are similar to each other.

$T$ and $S$ Points. For the $T(0, \pi, \pi)$ and 


\section{T. Kitagawa and T. Miyazawa}

$S(\pi, 0, \pi)$ points, the $\boldsymbol{k}$ group is $\mathrm{D}_{2 h}$ and irreducible representations are two-dimensional. Crystal vibrations of the $T$ point are doubly degenerate (Figure 3f). However, for the $S$ point, the time-reversal degeneracy appears and crystal vibrations of the $S$ point are quadruply degenerate (Figure 3e).

$H, C, E$ and $Q$ Points. For the $H\left(0, \delta_{b}, \pi\right)$, $C\left(\delta_{a}, 0, \pi\right), E\left(\delta_{a}, \pi, \pi\right)$ and $Q\left(\pi, \delta_{b}, \pi\right)$ points, the $\boldsymbol{k}$ group is $C_{2 v}$. Two-fold degeneracy of vibrations is due to the space symmetry $(H$ and $C)$ or to the time-reversal symmetry $(E)$ (Figures $3 \mathrm{~g}$ and $\mathrm{h}$ ). Dispersion curves of the $Q$ points are quadruply degenerate for any value of $\delta_{b}$, owing to the space and time-reversal degeneracies (Figure $3 \mathrm{~h}$ ). Crystal vibrations of the $H$, $C, E$ and $Q$ points are associated with the phase difference of $\delta_{c}=\pi$ and the vibrational frequencies change little with the phase differences of $\delta_{a}$ and $\delta_{b}$.

\section{FREQUENCY DISTRIBUTION}

Under the cyclic boundary condition, phasedifference vectors are distributed uniformly in the first Brillouin zone. However, for orthorhombic crystals with the factor group of $\mathrm{D}_{2 h}$, the irreducible volume is one-eighth of the first Brillouin zone. Vibrational frequencies of chain-polymer crystals change sensitively with the phase-difference $\left(\delta_{c}\right)$ along the chain axis but little with $\delta_{a}$ or $\delta_{b}$. Accordingly, for perdeuterated polyethylene, the value of $\delta_{a}$ or

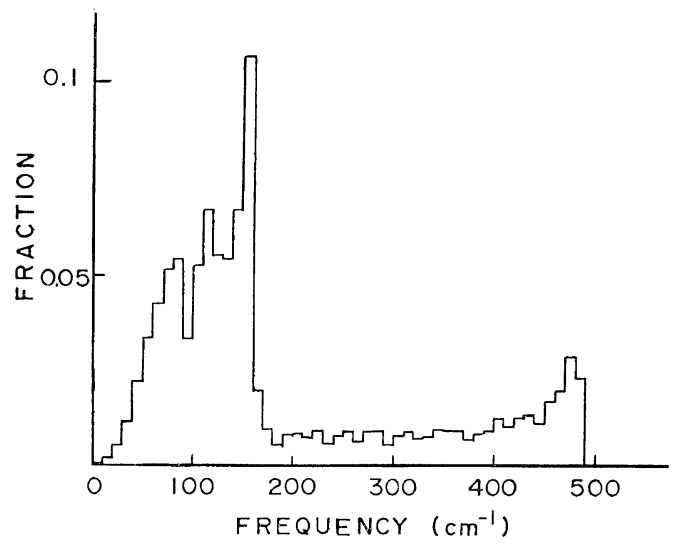

Figure 4. Frequency distribution of the orthorhombic form of perdeuterated polyethylene. $\delta_{b}$ was taken as $10,30,50, \ldots, 150$ or $170^{\circ}$ while the value of $\delta_{c}$ was taken as $0.5,1.5,2.5$, $\ldots, 178.5$ or $179.5^{\circ}$. Thus for a total of 14580 phase-difference vectors in the irreducible volume, 116,640 vibrations lying below $500 \mathrm{~cm}^{-1}$ were collected in a histogram of frequency distribution (Figure 4, frequency interval of $10 \mathrm{~cm}^{-1}$ ).

The distribution peak at $480 \mathrm{~cm}^{-1}$ corresponds to the maximum frequency of $\mathrm{C}-\mathrm{C}-\mathrm{C}$ bending vibrations (Figure 3). The prominent peak at $160 \mathrm{~cm}^{-1}$ is due to internal-rotation modes about $\mathrm{C}-\mathrm{C}$ bonds, while the peak at $120 \mathrm{~cm}^{-1}$ is due to overall rotatory modes $\left(R_{c}\right)$ about the chain axis. These distribution peaks correspond to the inelastic scattering peaks of thermal neutrons ${ }^{7}$ at 440,160 and $110 \mathrm{~cm}^{-1}$. The distribution peak at $75 \mathrm{~cm}^{-1}$ is due to translatory modes $\left(T_{a}\right.$ and $T_{b}$ ) perpendicular to the chain axis. The weak scattering peak observed ${ }^{7}$ near $250 \mathrm{~cm}^{-1}$ does not correspond to any distribution peak (Figure 4) and is not due to the one-phonon process. This scattering peak is possibly due to the two phonon process $\left[160 \mathrm{~cm}^{-1}\left(R_{c}\right)+\right.$ $75 \mathrm{~cm}^{-1}\left(T_{a}\right.$ and $\left.\left.T_{b}\right)\right]$.

\section{REFERENCES}

1. T. Kitagawa and T. Miyazawa, Bull. Chem. Soc. Japan, 42, 3437 (1969).

2. T. Kitagawa and T. Miyazawa, Bull. Chem. Soc. Japan, 43, 372 (1970).

3. W. Myers, G. C. Summerfield, and J. S. King, J. Chem. Phys., 44, 184 (1966).

4. T. Kitagawa and T. Miyazawa, Rept. Prog. Polym. Phys. Japan, 11, 219 (1968).

5. T. Kitagawa and T. Miyazawa, J. Chem. Phys., 47, 337 (1967).

6. T. Kitagawa and T. Miyazawa, J. Polym. Sci., Part B, 6, 83 (1968).

7. J. E. Lynch, Jr., G. C. Summerfield, L. A. Feldkamp, and J. S. King, J. Chem. Phys., 48, 912 (1968).

8. L. A. Feldkamp, G. Venkataraman, and J.S. King, "Neutron Inelastic Scattering", IAEA Symposium, Copenhagen, Vol. 2, 1968, p 159.

9. C. W. Bunn, Trans. Faraday Soc., 35, 482 (1939).

10. A. E. Smith, J. Chem. Phys., 21, 2229 (1953).

11. H. M. Shearer and V. Vand, Acta Cryst., 9, 379 (1956).

12. P. W. Teare, Acta Cryst., 12, 294 (1959). 
Crystal Vibrations and Dispersion Curves of Perdeuterated Polyethylene

13. R. G. Snyder, J. Mol.Spectry., 4, 411 (1960); 7, 116 (1961).

14. P. R. Swan, J. Polym. Sci., 56, 403 (1962).

15. J. H. Schachtschneider and R. G. Snyder, Spectrochim. Acta, 19, 117 (1963).

16. B. Wunderlich, J. Chem. Phys., 37, 1203, 1207 (1962).

17. M. Tasumi and T. Shimanouchi, J. Chem. Phys., 43, 1245 (1965).

18. J. C. Slater, "Quantum Theory of Molecules and Solids", Vol. 2, McGraw-Hill Company, New York, N.Y., 1965, p 118.
19. T. Kitagawa, "Crystal Dynamics and Solid State Properties of Polymers', Dissertation, Osaka University, Osaka, 1968.

20. L. P. Bouckaert, R. Smoluchowski, and E. Wigner, Phys. Rev., 50,58 (1936).

21. S. Krimm and M. I. Bank, J. Chem. Phys., 42, 4059 (1965); M. I. Bank and S. Krimm, J. Appl. Phys., 39, 4951 (1968).

22. M. Tasumi and S. Krimm, J. Chem. Phys., 45, 755 (1967).

23. R.F. Schaufele and T. Shimanouchi, J. Chem. Phys., 47, 3605 (1967). 